\title{
Three-Dimensional Visualization of Suprarenal Aortic Stent-Grafts: Evaluation of Migration in Midterm Follow-up
}

\section{Zhonghua Sun, PhD}

Department of Medical Imaging Science, Curtin University of Technology, Perth, Western Australia.

\begin{abstract}
৬>
Purpose: To investigate the midterm results of transrenal fixation of abdominal aortic stentgrafts with regard to device migration and encroachment of stent wires on the renal and visceral branches.

Methods: Imaging data from 18 patients (15 men; mean age 75 years, range 63-84) undergoing transrenal stent-graft fixation for abdominal aortic aneurysm (AAA) were included in the study. Computed tomographic angiographic data acquired within 1 week of stentgraft implantation were compared to the latest follow-up images. Postprocessing methods generated 3-dimensional (3D) maximum intensity projections (MIP) and virtual intravascular endoscopy (VIE) for evaluation of the relationship between suprarenal stents and aortic branches. Aortic neck angulation was measured in each patient for correlation with the incidence of stent migration.

Results: The mean follow-up period was 40 months. 3D image visualizations showed that the stent-graft moved caudally in all patients (range 2.6-14.2 mm), with migration $(>10$ $\mathrm{mm}$ ) observed in $4(22 \%)$ patients. Corresponding VIE images documented changes in stent wire encroachment on the aortic branch ostia in 11 patients, including the number and position of crossing stent wires. There was no close relationship between aortic neck angulation and stent migration.

Conclusion: The current study demonstrated that migration occurs at midterm follow-up in transrenally deployed stent-grafts. 3D images were valuable for the assessment of stent migration, as well as its relationship with aortic branch ostia. Long-term follow-up of transrenal fixation deserves to be investigated, especially after observing stent migration relative to aortic ostial encroachment.
\end{abstract}

J Endovasc Ther 2006;13:85-93

Key words: Stent-graft, computed tomography, 3D reconstruction, aortic ostium, suprarenal fixation, migration

Endovascular aneurysm repair has been widely used in clinical practice to treat patients with abdominal aortic aneurysm (AAA); this effective alternative to conventional open surgery has proven particularly advantageous in patients with comorbid medical conditions. ${ }^{1-3}$ Transrenal fixation of aortic stent- grafts, a modification of the commonly used infrarenal fixation, evolved to establish more secure proximal fixation in patients with unfavorable proximal neck anatomy, thereby expanding the applicability of endovascular repair to a wider group of patients..$^{4,5}$

Although early experience with transrenal

The author has no commercial, proprietary, or financial interest in any products or companies described in this article.

Address for correspondence and reprints: Dr. Zhonghua Sun, Department of Medical Imaging Science, Curtin University of Technology, GPO Box, U1987, Perth, Western Australia 6845, Australia. Fax: 61-8-9266-4344; E-mail: z.sun@curtin. edu.au 
fixation of aortic stent-grafts seemed promising and short to midterm results were satisfactory, ${ }^{6-10}$ long-term effects are still not fully understood. The long-term outcomes of transrenal AAA repair can be determined from the late complications, such as endoleaks, renal dysfunction, stent-graft occlusion, aneurysm rupture, and stent-graft migration. Of these complications, device migration is a challenging one, posing a significant risk for aneurysm rupture. ${ }^{11}$

Theoretically, transrenal fixation secures proximal fixation and prevents endograft migration by placing stents across the renal and other visceral ostia. ${ }^{12-14}$ This technique should reduce the incidence of stent migration because it is deployed in the less severely diseased aorta, which is less likely to demonstrate significant growth compared to the infrarenal aortic neck. ${ }^{15}$ Because studies performed have shown that stent barbs and hooks increase fixation compared to infrarenal deployment, 16,17 transrenal fixation has added hooks and barbs to increase proximal attachment. However, few studies have focused on stent-graft migration after endovascular repair, especially in stent-grafts with the transrenal fixation. ${ }^{18-20}$ Moreover, quantitative assessment of stent migration is not routinely performed.

This study was designed to investigate stent-graft migration, as well as its relationship to the aortic branch ostia, using 3-dimensional (3D) computed tomography (CT). Helical CT angiography (CTA) has been reported as the preferred modality for both pre and post stent-graft imaging, ${ }^{21,22}$ and 3D CT reconstructions were found to provide additional information compared to conventional 2D images. ${ }^{23,24}$ Therefore, in this data analysis, 3D image visualizations were used to assess both stent migration and encroachment of stent wires on the aortic branch ostia.

\section{METHODS}

\section{Patient Sample and Image Acquisition}

Imaging data from 18 patients ( 15 men; mean age 75 years, range $63-84$ ) undergoing endovascular AAA repair were included in the study. All patients were treated with the Ze- nith AAA Endovascular Graft (William Cook Europe, Bjaeverskov, Denmark) with an uncovered component placed above the renal arteries for proximal fixation.

Helical CTA was performed in each patient on a single-slice CT scanner (Philips AV-E1; Philips Medical Systems, Inc., Shelton, CT, USA) within 1 week of the stent-graft implantation. In the more recent surveillance imaging, a multislice CT scanner (Toshiba Medical Systems Europe, Zoetermeer, The Netherlands) was used. The scanning protocol for single-slice CTA was $5-\mathrm{mm}$ collimation, pitch 1.0, and a $2-\mathrm{mm}$ reconstruction interval with a 1-second gantry rotation time. For the multislice CTA, the section thickness was $1 \mathrm{~mm}$ (16×1-mm beam collimation), pitch 2.0, 1-mm reconstruction interval, and 0.5 -second gantry rotation time. Both single and multislice CTA scans were performed with an intravenous injection of $100 \mathrm{~mL}$ of nonionic contrast media (Niopam 300; Bracco UK Ltd., High Wycombe, UK) administered at a rate of $2 \mathrm{~mL} / \mathrm{s}$ with a scan delay of 30 seconds. Therefore, all 18 patients had a mixture of single and multislice scans in their follow-up.

\section{D Image Postprocessing}

All CT datasets were transferred to a separate workstation for generation of 3D images and measurements. CT volume data were converted from original DICOM (Digital Imaging and Communication in Medicine) images with commercially available software (Analyze V 5.0; AnalyzeDirect, Inc., Lenexa, KS, USA).

3D postprocessing reconstructions, including maximum intensity projections (MIP) and virtual intravascular endoscopy (VIE), were performed to demonstrate stent-graft position relative to the arterial branches at follow-up. Details of 3D image generation have been described elsewhere. ${ }^{23}$ A series of MIP views were created to measure stent migration, while VIE images were used to determine if there was any change in configuration of stent wires in relation to the aortic branch ostia. 


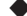

TABLE

3D Visualization of Stent-Graft Migration and Stent Wires Relative to the Aortic Branch Ostia

\begin{tabular}{|c|c|c|c|c|c|c|c|c|c|c|c|}
\hline \multirow[b]{2}{*}{ Pt. } & \multirow{2}{*}{$\begin{array}{c}\text { Neck } \\
\text { Angulation } \\
\text { Change, }^{\circ}\end{array}$} & \multirow{2}{*}{$\begin{array}{c}\text { Caudal Stent } \\
\text { Migration, } \\
\text { mm }\end{array}$} & \multicolumn{4}{|c|}{ Stent Wires at Initial Imaging } & \multicolumn{4}{|c|}{ Stent Wires at Latest Follow-up } & \multirow[b]{2}{*}{$\begin{array}{c}\text { Follow-up, } \\
\text { mo }\end{array}$} \\
\hline & & & Celiac & SMA & RRA & LRA & Celiac & SMA & RRA & LRA & \\
\hline 1 & -1.3 & 4.5 & - & $M$ & M & M & - & M & $M$ & M & 48 \\
\hline 2 & -16.8 & 9.3 & - & - & M & M & - & - & $M$ & $\mathrm{~S}$ & 36 \\
\hline 3 & -7.0 & 5.4 & - & M & M & $\mathrm{S}$ & - & M & M & M & 36 \\
\hline 4 & -4.2 & 8.1 & M & $\mathrm{S}$ & M & M & - & M & M & M & 36 \\
\hline 5 & -7.5 & 11.1 & - & $\mathrm{S}$ & $\mathrm{S}$ & $M$ & - & $\mathrm{S}$ & $M$ & $M$ & 42 \\
\hline 6 & +5.5 & 2.8 & - & $M$ & $M$ & $M$ & - & $M$ & $M$ & $M$ & 48 \\
\hline 7 & -0.4 & 5.0 & - & $S$ & $S$ & $M$ & - & $S$ & $\mathrm{~S}$ & $M$ & 36 \\
\hline 8 & -10.1 & 10.2 & - & $M$ & $\mathrm{~S}$ & $M$ & - & $\mathrm{S}$ & $S$ & $M$ & 24 \\
\hline 9 & 0 & 14.2 & - & $\mathrm{M}$ & $M$ & $S$ & - & - & $\mathrm{S}$ & $\mathrm{S}$ & 48 \\
\hline 10 & +4.3 & 4.2 & - & $M$ & - & $M$ & - & $M$ & - & $M$ & 54 \\
\hline 11 & +6.7 & 2.6 & - & $\mathrm{S}$ & $\mathrm{S}$ & $\mathrm{S}$ & - & $\mathrm{S}$ & $S$ & $\mathrm{~S}$ & 48 \\
\hline 12 & -25.4 & 10.2 & - & $M$ & $\mathrm{~S}$ & $M$ & - & $M$ & $M$ & $M$ & 36 \\
\hline 13 & -1.8 & 5.7 & - & - & $\mathrm{S}$ & $\mathrm{S}$ & - & - & $\mathrm{S}$ & $\mathrm{S}$ & 48 \\
\hline 14 & -4.6 & 3.5 & - & $M$ & $M$ & $\mathrm{~S}$ & - & $S$ & $M$ & $M$ & 36 \\
\hline 15 & -1.6 & 7.8 & $M$ & $\mathrm{M}$ & $M$ & - & $M$ & $M$ & $\mathrm{~S}$ & - & 36 \\
\hline 16 & -18.8 & 3.0 & $\mathrm{~S}$ & $\mathrm{M}$ & $M$ & $\mathrm{~S}$ & - & $M$ & $M$ & $M$ & 36 \\
\hline 17 & +3.5 & 6.7 & - & $\mathrm{M}$ & $M$ & $M$ & - & $M$ & $M$ & $M$ & 48 \\
\hline 18 & -4.8 & 7.4 & - & $\mathrm{M}$ & $M$ & $S$ & - & - & $M$ & $M$ & 36 \\
\hline
\end{tabular}

SMA: superior mesenteric artery, RRA: right renal artery, LRA: left renal artery, S: single stent wire, M: multiple stent wires, VIE: virtual intravascular endoscopy.

\section{Image Assessment}

As most aortic stent-grafts were deployed above the superior mesenteric artery (SMA), migration of the stent-grafts was determined by measuring the distance between the celiac axis or SMA and the most cephalad portion of the stent seen on the sagittal MIP images. Stent migration was defined as caudal device displacement $>10 \mathrm{~mm}$ from the previous CT studies. Aortic neck angulation was also measured from the first and the most recent follow-up data to investigate if there was a relationship to stent-graft migration. The encroachment of stent wires on the aortic branch ostia and the number of stent wires crossing the branches were assessed on VIE images to determine if a correlation existed with stent migration.

Maximal transverse AAA diameter was measured at 1, 3, 6, and 12 months then annually to monitor the change in the aneurysm sac. Renal function was evaluated using serum creatinine levels, which were measured preoperatively, prior to discharge, and at regular follow-up examinations. A Student $t$ test was used to analyze comparisons; $p<0.05$ was taken to indicate a statistically significant difference.

\section{RESULTS}

The follow-up time ranged from 24 to 54 months (mean 40.0 \pm 7.6 ). The Table displays the data on stent migration and encroachment of stent wires on the aortic branch ostia at the first and last imaging session. Caudal stent-graft movement was noticed in all patients to a variable extent, ranging from 2.6 to $14.2 \mathrm{~mm}$ (mean $6.2 \pm 3.3$ ). Four (22\%) of 18 patients showed stent migration on CTA, with displaced distance ranging from 10.2 to 14.2 $\mathrm{mm}$ (mean 11.4 \pm 1.9 ). The ranges of aneurysm neck angulation measurements were $0^{\circ}$ to $33.7^{\circ}$ at the first imaging study and $0^{\circ}$ to $28^{\circ}$ at the most recent postoperative followup. Neck angulation decreased in 13 (72\%) patients, increased in $4(22 \%)$ patients, and remained unchanged in $1(6 \%)$ patient. Although stent migration did occur in the angulated aneurysm necks, there was no direct relationship between stent migration and the degree of neck angulation (Table). 


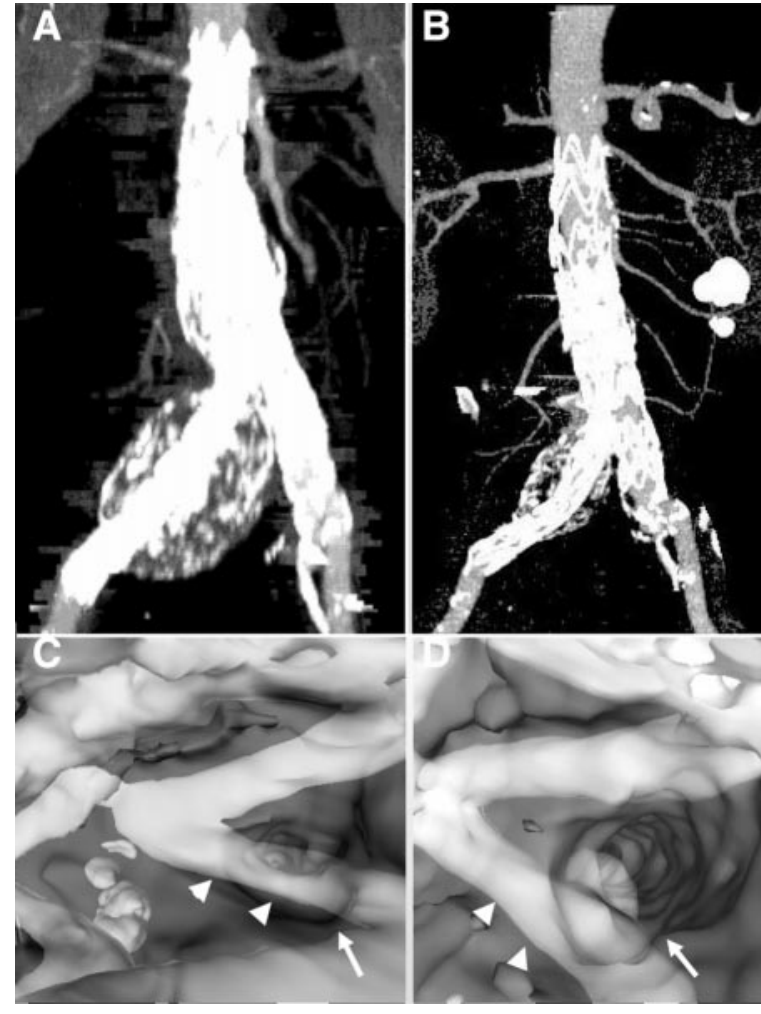

Figure $1 \diamond$ A 70-year-old man with an abdominal aortic aneurysm was treated with a suprarenal stent-graft. Helical CT images were acquired after the implantation (A) and at 48-month follow-up (B). The stent-graft did not show significant migration on the coronal CT MIP images; the corresponding VIE (C, D) showed that the left renal ostium (arrow) was crossed by a single stent wire (arrowheads) with nearly the same configuration.

The 3D relationship of stent wires relative to the aortic branch ostia was clearly visualized on VIE images (Figs. 1 and 2). Changes in configuration of the wires were noted in 11 $(61 \%)$ cases. The celiac ostium was crossed by one or more stent wires in 3 cases on the postoperative images, but in the latest followup, 2 of them were no longer covered due to distal movement of stent-grafts. In contrast, 16 SMA ostia were crossed by stent wires in the initial follow-up, and 2 of them were free of encroachment on the latest images. Bilateral renal ostia were crossed by stent wires in both sets of images; however, the configuration of stent wires relative to the renal ostia changed in 9 of 18 cases.

Over the mean 40-month follow-up, the aor-

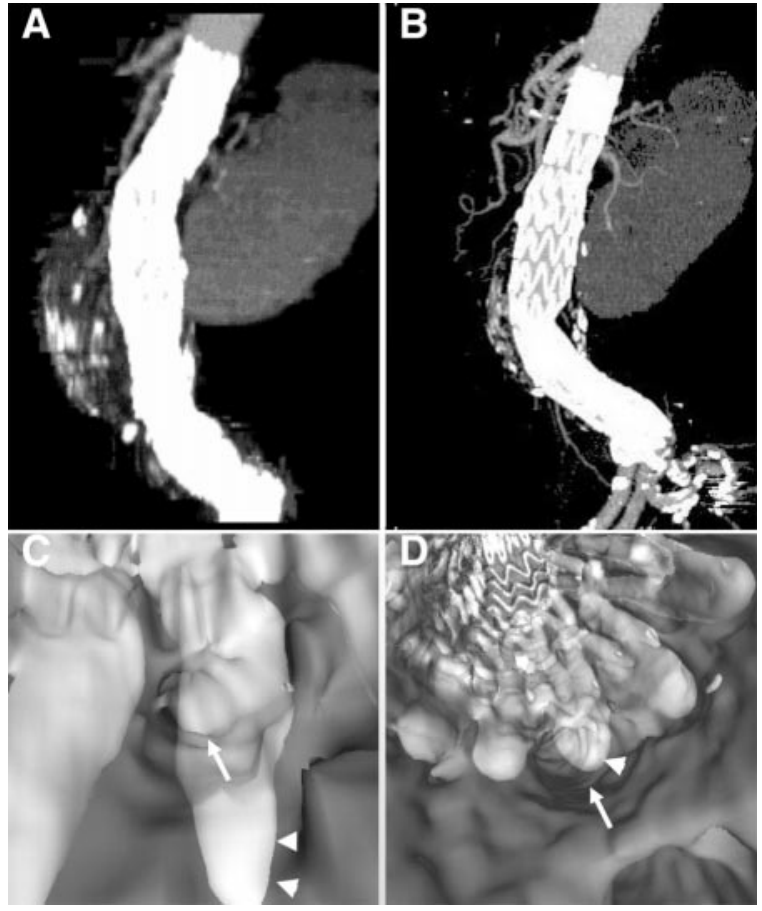

Figure $2 \diamond$ An 81-year-old woman with an AAA was treated with a suprarenal stent-graft (A) and evaluated at 36 months. Stent migration of $10.2 \mathrm{~mm}$ was noted in the most recent CT MIP image (B). VIE (C) at 1 week post implantation showed that a stent wire was encroaching on the SMA ostium (arrow), but at 36 months (D), the stent wires (arrowheads) had shifted.

tic aneurysms showed continuous shrinkage in $17(94 \%)$ patients, with a reduction in the maximal transverse diameter ranging from 2 to $32 \mathrm{~mm}$ (mean 13.8 \pm 7.5 ). In the remaining patient, the aneurysm had expanded from 62 to $76 \mathrm{~mm}$; a type I endoleak was found and embolized. The stent-graft migrated $7.8 \mathrm{~mm}$ caudally in this patient, which was not significant. Five endoleaks were found: types I and III in 1 patient and type II in 3 cases. Renal function was not significantly affected ( $p>0.05$ ) except in 1 patient who developed chronic renal failure due to an atrophic left renal artery and received dialysis.

\section{DISCUSSION}

One of the key points for successful endovascular repair requires secure proximal fixation to prevent stent-graft migration and avoid proximal endoleak. Because positional stabil- 
ity of the stent-graft is essential for long-term durability, proximal stent migration is another important potential failure that needs the attention of clinicians. ${ }^{25,26}$

There are two possible reasons for stent migration: (1) an increase in the aneurysm neck diameter over time, which decreases friction between the stent-graft and the aortic wall; and (2) the characteristics of proximal fixation. ${ }^{27}$ The first situation occurs in both infrarenally and suprarenally fixed stent-grafts because they use radial force to create wall friction that contributes to stability within the aorta. However, Wever et al. ${ }^{15}$ reported that the infrarenal aortic neck demonstrated continued dilatation while the dimensions of the suprarenal aorta did not change.

As regards fixation techniques, transrenal fixation has been shown to reduce the incidence of stent migration compared to infrarenally fixed stent-grafts. ${ }^{4}$ The deployment of aortic stent-grafts in relatively normal or less severely diseased suprarenal aorta provides an anchoring zone for proximal fixation.

The incidence of stent migration varies considerably among studies, with a range between $5 \%$ and $45 \% .{ }^{18,19}$ Different definitions of stent migration, varying designs of the stent-grafts, and the length of follow-up may explain this wide interval. Clinical studies have shown that stent migration occurred more frequently in infrarenal fixation than in the transrenal group. ${ }^{28,29}$ Conners et al. ${ }^{28}$ evaluated stent migration from 1 to 4 years based on single-center data from 91 patients with infrarenal stent-graft fixation. They noted a cumulative migration rate of $7.2 \%$ at 1 year, $20.4 \%$ at 2 years, $42.1 \%$ at 3 years, and $66.7 \%$ at 4 years after implantation. Their results illustrated that the AneuRx device without fixation is not suitable for complicated or hostile necks. Similarly, Cao et al. ${ }^{20}$ showed that $27 \%$ of their 113 patients undergoing infrarenal AAA repair with the AneuRx device had stent migration at 3 years; they concluded that stent migration is a frequent event that requires the surgeon to make a therapeutic decision. Kalliafas et al. ${ }^{17}$ reported on a series of 176 patients treated with their Nottingham system; the rate of graft migration in infrarenally fixed grafts was $10.9 \%$ compared to the $2.1 \%$ observed in suprarenally fixed grafts.
At a mean follow-up of 40 months, our study showed that $22 \%$ of AAA patients treated with Zenith stent-grafts had stent migration, which was more frequent than reported by others. ${ }^{17,30,31}$ It is not clear why we had such a high incidence of stent migration despite the decreased neck angulation in our patients. One possible explanation may be that our technique of measurement, combining 3D MIP and VIE images, is superior to that employed by others who used 2D axial CT or multiplanar reformatted images. The pathophysiology of stent-graft migration is complex, and etiologies of stent-graft migration are likely to be multifactorial, as some patients with severe angulated necks did not have the significant migration observed in other studies. ${ }^{4,28}$

Accurate measurement of stent migration is important, and we consider it necessary to perform the measurements based on 3D reconstructions. It is difficult to define the distance of migration on 2D axial CT images, so under or overestimation of migration distance would be unavoidable. Based on our experience, measurement of stent migration was successfully performed on MIP images. Moreover, comparison with previous 3D images allowed the reader to visualize not only the location of stent wires but also their relationship to the aortic branches.

Previous studies have shown that VIE images were valuable for visualization of stent wire encroachment on aortic branch ostia and assessment of the effect of transrenal fixation. ${ }^{23,24}$ The current study further supported the observation that stent migration results in subsequent configuration changes in stent wires relative to the aortic branch ostia, including a change in the number of stent wires crossing the ostia. Of 4 patients with stent migration in our study, VIE images clearly showed that the configuration of stent wires relative to aortic branch ostia was altered due to migration.

As the long-term effect of transrenal fixation is unclear, the additional information provided by VIE would be valuable to surgeons when assessing the outcomes of transrenal fixation in the following areas. First, potential interference with renal blood flow could be assigned to stent wires encroaching on renal 


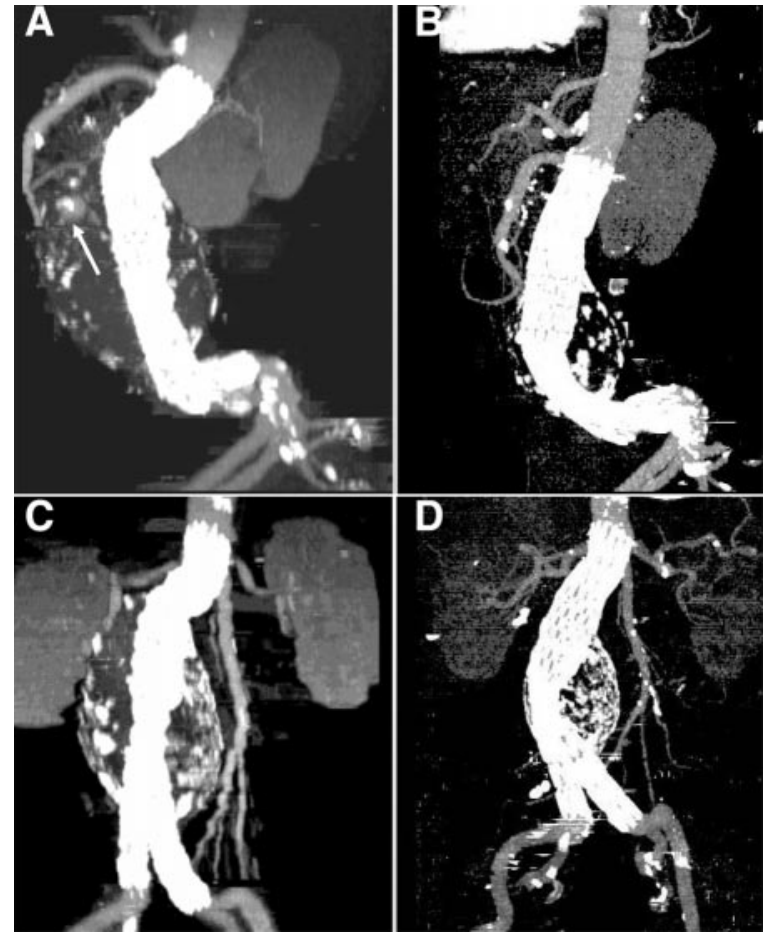

Figure $3 \bullet$ Two AAA patients undergoing transrenal fixation developed stent-graft distortion leading to stent migration. (A) A 74-year old man had stent migration of $10.2 \mathrm{~mm}$ due to foreshortening of the longitudinal aneurysm sac at the 24-month followup (B). The arrow in A indicates a type II endoleak, which resolved spontaneously. (C) A 75-year-old man developed stent migration of $11.1 \mathrm{~mm}$ at 36month follow-up (D) due to distortion of stent-graft.

artery ostia, which might alter hemodynamics and lead to the dispersion of late multiple emboli. ${ }^{32}$ Second, any reduction in cross-sectional area of the aortic branch ostia could be caused by stent wires ${ }^{12,13}$; this scenario could alter renal perfusion and affect renal function.

The diameter of aortic stent wires in our study was $0.4 \mathrm{~mm}$, while diameters of aortic branch ostia ranged from 5 to $10 \mathrm{~mm}$. Therefore, reduction of the ostial cross-sectional area was $<20 \%$ based on our previous results from an in vitro phantom study. ${ }^{13}$ However, the stent wire thickness was overestimated on CT images (2 to $3 \mathrm{~mm}$ in diameter), which made it appear that nearly $50 \%$ of the renal artery ostium was covered (Figs. 1 and 2). Although the measured thickness does not reflect the real size of a stent wire, there is a possibility of neointimal hyperplasia forming over the stent wires, which might further decrease the cross-sectional area of branch ostia. ${ }^{33}$ We believe that the VIE findings presented in this study will aid vascular surgeons in accurately assessing the effect of transrenal fixation and therapeutic management. Thus, we recommend that VIE be used as a routine technique in AAA patients treated with transrenal fixation.

The Zenith stent-graft used in our study, which has $25-\mathrm{mm}$-long uncovered suprarenal stent struts, employs active fixation with hooks that are placed above the renal arteries. However, active fixation at the proximal attachment system in patients with hostile aneurysm neck (severely angulated, short, thrombus-laden, or morphologically complex) is reported to carry some risks, such as extravasation or pseudoaneurysm at the site of proximal fixation. ${ }^{28}$ With $3 \mathrm{D}$ images, we did not observe any of these complications in our small group, but the potential danger of stent struts crossing the renal arteries should not be ignored by surgeons from a long-term point of view. $13,14,34$

It is possible that the morphology of the aneurysm sac and adjacent aortic branches changes following endovascular AAA repair. In some cases, foreshortening of the sac reduces the distance between the proximal and distal anchor sites, causing the stent-graft to buckle, kink, migrate, or occlude a graft limb. ${ }^{35}$ Our results showed longitudinal foreshortening of aneurysm sac in 2 of 4 patients with stent migration (Fig. 3), which resulted in subsequent distortion of the stent-grafts. It seems likely that this structural alteration may be a contributing factor to our high late complication rate. Although neck angulation decreased in most cases in our study, 4 of 18 patients showed increased neck angles following transrenal fixation. There might be a relationship between the aneurysm size and neck angulation, as 3 of these 4 patients showing increased neck angles had an aneurysm $>6 \mathrm{~cm}$. A robust conclusion cannot be drawn from our small cohort, but it would be worthwhile investigating the relationship between the incidence of stent-graft distortion and stent migration in a large cohort. 

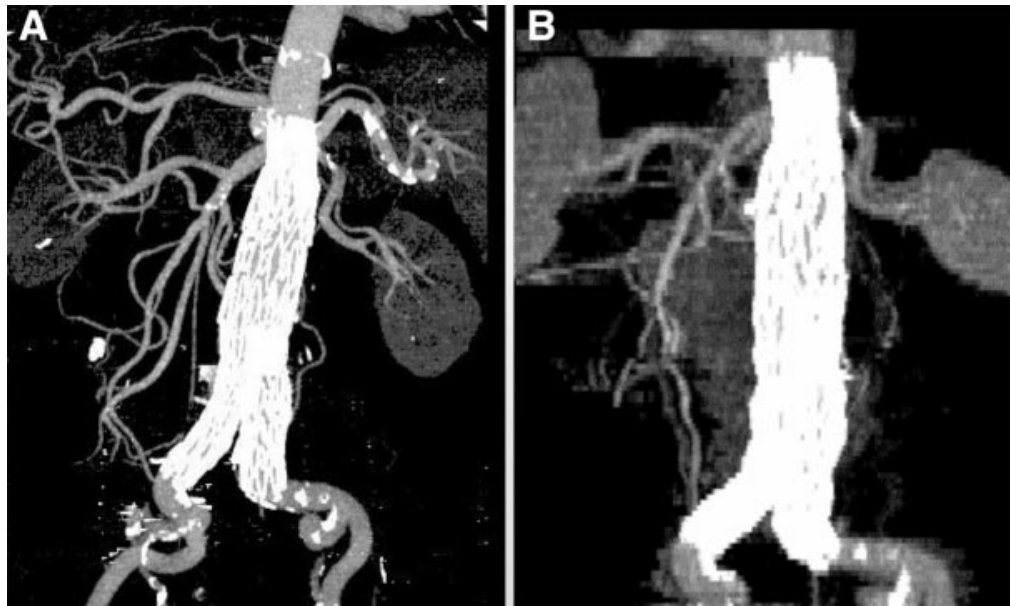

Figure $4 \rightarrow 3 D$ visualization of the aortic branches and the stent-grafts was significantly improved in data acquired with multislice CT (A) compared to those from the single-slice CT (B).

\section{Limitations}

Our study consisted of a small number of patients whose imaging data were examined at only 2 time points, not serially over the follow-up period. Comparison of annual CT data, for example, may help us determine the prevalence of stent migration as well as its relationship to changes in the aneurysm shape or neck angulation. Although VIE was successfully generated in all patients to demonstrate the intraluminal stent wires relative to aortic branch ostia, image quality was affected by the presence of stair-step artifacts in some patients. ${ }^{36}$ This problem was especially apparent in the first postoperative group because the data were acquired with single-slice CT scans at $5-\mathrm{mm}$ slice thickness and 2- $\mathrm{mm}$ reconstruction intervals. Visualization of aortic branches and stent wires was significantly improved in the most recent multislice CT scans (Fig. 4), which were performed with thinner sections and reconstruction intervals. Because multislice CT can acquire nearly isotropic volume data, ${ }^{37,38}$ it clearly reveals the abdominal aorta, its branches, and the stent wires better than single-slice CT (Fig 4). Consequently, the quality of VIE images was improved with multislice CT scanning. Addressing the treatment options for stent migration was beyond the scope of this study and has been discussed by others. ${ }^{20,27}$

\section{Conclusion}

Our study showed that migration does occur in stent-grafts with transrenal fixation at midterm follow-up. Stent migration also results in subsequent configuration changes in stent wires relative to the aortic branch ostia, which is best visualized and assessed on 3D image reconstructions.

3D VIE images clearly showed the changes in the relationship between stent wires and aortic branch ostia due to stent migration in AAA patients treated with suprarenal stentgrafts. Additional intraluminal information provided by VIE will aid vascular surgeons in accurately assessing the interference of stent wires with renal artery ostia. Future study in large groups should be performed to investigate the long-term stability of transrenal stent-graft fixation.

Acknowledgments: I would like to thank Dr. Peter Ellis and Mr. Mark O'Donnell for their assistance in data transfer and clinical cooperation.

\section{REFERENCES}

1. Buth J, van Marrewijk CJ, Harris PL, et al. Outcome of endovascular abdominal aortic aneurysm repair in patients with conditions considered unfit for an open procedure: a report on the EUROSTAR experience. J Vasc Surg. 2002; 35:211-221.

2. Cao P, Verzini F, Parlani G, et al. Clinical effect 
of abdominal aortic aneurysm endografting: 7year concurrent comparison with open repair. $J$ Vasc Surg. 2004;40:841-848.

3. Prinssen M, Verhoeven EL, Buth J, et al. A randomized trial comparing conventional and endovascular repair of abdominal aortic aneurysms. N Engl J Med. 2004;351:1607-1618.

4. Robbins M, Kritpracha B, Beebe HG, et al. Suprarenal endograft fixation avoids adverse outcomes associated with aortic neck angulation. Ann Vasc Surg. 2005;19:172-177.

5. Lau LL, Hakaim AG, Oldenburg WA, et al. Effect of suprarenal versus infrarenal aortic endograft fixation on renal function and renal artery patency: a comparative study with intermediate follow-up. J Vasc Surg. 2003;37:1162-1168.

6. Malina M, Lindh M, Ivancev $K$, et al. The effect of endovascular aortic stents placed across the renal arteries. Eur J Vasc Endovasc Surg. 1997; 13:207-213.

7. Ferko A, Krajina A, Jon B, et al. Juxtarenal aortic aneurysm: endoluminal transfemoral repair? Eur Radiol. 1997;7:703-707.

8. Lobato AC, Quick RC, Vaughn PL, et al. Transrenal fixation of aortic endografts: intermediate follow-up of a single-center experience. J Endovasc Ther. 2000;7:273-278.

9. Bove PG, Long GW, Zelenock GB, et al. Transrenal fixation of aortic stent-grafts for the treatment of infrarenal aortic aneurysmal disease. $J$ Vasc Surg. 2000;32:697-703.

10. Alric P, Hinchliffe RJ, Picot MC, et al. Long-term renal function following endovascular aneurysm repair with infrarenal and suprarenal aortic stent-grafts. J Endovasc Ther. 2003;10:397405.

11. Zarins CK, White RA, Fogarty TJ. Aneurysm rupture after endovascular repair using the AneuRx stent graft. J Vasc Surg. 2000;31:960970.

12. Liffman K, Lawrence-Brown MMD, Semmens $\mathrm{JB}$, et al. Suprarenal fixation: effect on blood flow of an endoluminal stent wire across an arterial orifice. J Endovasc Ther. 2003;10:260274.

13. Sun Z, Zheng H. Cross-sectional area reduction of the renal ostium by suprarenal stent wires: in vitro phantom study by CT virtual angioscopy. Comput Med Imaging Graph. 2004;28: 345-351.

14. Sun Z. Transrenal fixation of aortic stent-grafts: current status and future directions. $J$ Endovasc Ther. 2004;11:539-549.

15. Wever JJ, de Nie AJ, Blankensteijn JD, et al. Dilatation of the proximal neck of infrarenal aortic aneurysms after endovascular AAA re- pair. Eur J Vasc Endovasc Surg. 2000;19:197201.

16. Malina M, Lindblad B, Ivancev $K$, et al. Endovascular AAA exclusion: will stents with hooks and barbs prevent stent-graft migration? J Endovasc Surg. 1998;5:310-317.

17. Kalliafas S, Albertini JA, Macierewicz J, et al. Stent-graft migration after endovascular repair of abdominal aortic aneurysm. J Endovasc Ther. 2002;9:743-747.

18. Resch T, Ivancev K, Brunkwall J, et al. Distal migration of stent-grafts after endovascular repair of abdominal aortic aneurysms. J Vasc Interv Radiol. 1999;10:257-264.

19. Greenberg RK, Lawrence-Brown M, Bhandari $G$, et al. An update of the Zenith endovascular graft for abdominal aortic aneurysms: initial implantation and mid-term follow-up data. $J$ Vasc Surg. 2001;33:S157-164.

20. Cao P, Verzini F, Zannetti S, et al. Device migration after endoluminal abdominal aortic aneurysm repair: analysis of 113 cases with a minimum follow-up period of 2 years. J Vasc Surg. 2002;35:229-235.

21. Broeders IA, Blankensteijn JD, Olree $M$, et al. Preoperative sizing of grafts for transfemoral endovascular aneurysm management: a prospective comparative study of spiral CT angiography, arteriography, and conventional CT imaging. J Endovasc Surg. 1997;4:252-261.

22. Armerding MD, Rubin GD, Beaulieu CF, et al. Aortic aneurysmal disease: assessment of stent-graft treatment-CT versus conventional angiography. Radiology. 2000;215:138-146.

23. Sun Z, Winder J, Kelly B, et al. CT virtual intravascular endoscopy of abdominal aortic aneurysms treated with suprarenal endovascular stent-grafting. Abdom Imaging. 2003;28:580587.

24. Sun Z, Winder RJ, Kelly BE, et al. Diagnostic value of $C T$ virtual intravascular endoscopy in aortic stent-grafting. J Endovasc Ther. 2004;11: 13-25.

25. Albertini JN, Kalliafas S, Travis S, et al. Anatomical risk factors for proximal perigraft endoleak and graft migration following endovascular repair of abdominal aortic aneurysms. Eur J Vasc Endovasc Surg. 2000;19:308-312.

26. Greenberg RK, Turc A, Haulon S, et al. Stentgraft migration: a reappraisal of analysis methods and proposed revised definition. $J$ Endovasc Ther. 2004;11:353-363.

27. Towne JB. Endovascular treatment of abdominal aortic aneurysms. Am J Surg. 2005;18: 140-149.

28. Conners MS, Sternbergh WC, Carter G, et al. 
Endograft migration one to four years after endovascular abdominal aortic aneurysm repair with the AneuRx device: a cautionary note. $J$ Vasc Surg. 2002;36:476-484.

29. Dillavou ED, Muluk SC, Rhee RY, et al. Does hostile neck anatomy preclude successful endovascular aortic aneurysm repair? J Vasc Surg. 2003;38:657-663.

30. Greenberg RK, Chuter TA, Sternbergh WC, et al. Zenith AAA endovascular graft: intermediate-term results of the US multicenter trial. $J$ Vasc Surg. 2004;39:1209-1218.

31. Cowie AG, Ashleigh RJ, England RE, et al. Endovascular aneurysm repair with the Talent stent-graft. J Vasc Interv Radiol. 2003;14:10111016.

32. Chong CK, How TV. Flow patterns in an endovascular stent-graft for abdominal aortic aneurysm repair. J Biomech. 2004;37:89-97.

33. Desgranges $P$, Huntin E, Kedzia C, et al. Aortic stents covering the renal artery ostia: an animal study. J Vasc Interv Radiol. 1997;8:77-82.
34. Krämer SC, Görich J, Bachmann R, et al. Incidence of renal infarctions after transrenal stent placement in an animal model. $J$ Endovasc Ther. 2005;12:312-317.

35. Harris P, Brennan J, Martin J, et al. Longitudinal aneurysm shrinkage following endovascular aortic aneurysm repair: a source of intermediate and late complications. J Endovasc Surg. 1999;6:11-16.

36. Fleischmann D, Rubin GD, Paik DS, et al. Stairstep artifacts with single versus multiple detector-row helical CT. Radiology. 2000;216:185196.

37. Rubin GD, Shiau MC, Leung AN, et al. Aorta and iliac arteries: single versus multiple detector-row helical CT angiography. Radiology. 2000;215:670-676.

38. Rydberg J, Kopecky KK, Lalka SG, et al. Stentgrafting of abdominal aortic aneurysms: preand postoperative evaluation with multislice helical CT. J Comput Assist Tomogr. 2001;25: 580-586. 